\title{
Crítica constructiva a la asignatura de Ecuaciones Diferenciales, perteneciente al tercer semestre de la licenciatura en ingeniería industrial, impartida en una universidad del centro-sur del país
}

\section{Constructive criticizing to differential equations class, from the third semester of industrial engineering in a south-central Mexican university}

Héctor D. Molina-Ruiz ${ }^{a}$.

\begin{abstract}
:
Education in Mexico plays an important role in country's development. Considering that professionals graduated from public and private universities, will have in their hands the decision-making process in public and private organizations in Mexico and in the world, it is necessary that education undergoes a process of analysis, reflection and improvement. The above, by the objective of ensuring education's quality, taught by universities. This document constitutes a reflection essay on differential equations' class, belonging to third semester of an industrial engineering program, taught at a university in south-central Mexico.
\end{abstract}

Keywords:

Differential Equations, Improvement Proposal, Subject

\section{Resumen:}

La educación en México, juega un rol importante en el desarrollo del país. Considerando que los profesionistas egresados de la universidades públicas y privadas, tendrán en sus manos las decisiones de las organizaciones públicas y privadas en México y en el mundo, es necesario que la impartición de educación se someta a proceso de análisis, reflexión y mejora. Lo anterior, con el objetivo de asegurar la calidad de la educación, impartida por las universidades del país. El presente documento, constituye un ensayo de reflexión al respecto de la materia de ecuaciones diferenciales, perteneciente al tercer semestre de la licenciatura en ingeniería industrial, impartida en una universidad del centro-sur del país.

\section{Palabras Clave:}

Asignatura, Ecuaciones Diferenciales, Propuesta de Mejora

\section{Introducción}

Las matemáticas y sus diferentes ramas, han potenciado el desarrollo de la humanidad. Las matemáticas, han estado presentes en culturas y civilizaciones milenarias. Adhikari (2019) apunta que entre las civilizaciones más antiguas se encuentran: la Mesopotámica (3500 A.C.-500 A.C.), Indú (3300 A.C. - 1900 A.C.), Egipcía (3150 A.C. 30 D.C.), griega (2700 A.C. - 479 D.C.), Maya (2600 A.C. - 900 D.C.), China (1600 A.C. - 1046 A.C.), persa (550 A.C. - 331 D.C.), Romana (550 A.C. - 465 D.C.), Azteca (1345 D.C. - 1521 D.C.) e Inca (1438 D.C. - 1532 D.C.). Para Neugebauer (1961), parte de la ciencia antigua tiene su base en la transmisión del conocimiento em dos áreas básicas, las matemáticas y la astronomía. Por su parte Seidenberg (1978), apunta que existen dos grandes tradiciones, fácilmente discernibles, en la historia de las matemáticas: la geometría o constructiva y la algebraica o computacional.

Las matemáticas chinas empiezan a ser más detalladas a en el periodo de la dinastía HAN (208 A.C. - 8 D.C.), sin embargo, el corpus matemático de Egipto y Babilonia las preceden por más de un milenio (Martzloff, 1997). En Boyer \& Merzbach (2011), se expone que se encuentra registro del uso de fracciones para el antiguo Egipto desde el año 2000 A.C.

Una de las ramas importantes de las matemáticas, es el campo del cálculo diferencial e integral, en el cual se encuentran las ecuaciones diferenciales. Como se menciona en Quarteroni \& Saleri (2006), una ecuación diferencial es una ecuación que involucra una o más derivadas de una función desconocida, para la cual, si todas las derivadas se toman con respecto a una sola

Autor de Correspondencia, Universidad Autónoma del Estado de Hidalgo, https://orcid.org/0000-0003-4657-3237, Email: 
variable independiente se llama ecuación diferencial ordinaria, mientras que hablaremos de una ecuación en derivadas parciales cuando estén presentes derivadas parciales.

La materia de ecuaciones diferenciales, permite genera un proceso cognitivo para la toma de decisiones, dado que la asignatura cuenta con métodos y procedimientos para la solución de problemas, que se equiparan al proceso cognitivo usado al afrontar el mercado laboral, particularmente en el momento de la toma de decisiones.

\section{Crítica a la asignatura de ecuaciones diferenciales}

A continuación, se realiza un análisis de la asignatura y se hace una crítica con la consecuente propuesta de mejora para la impartición de la clase.

\subsection{Temas que conforman la asignatura}

La primera unidad denominada "1. Las Ecuaciones Diferenciales", sienta las bases para la compresión del contexto de la asignatura, además de propiciar el entendimiento de la relación que esta guarda con el proceso cognitivo de toma de decisiones, para el desarrollo profesional del estudiante.

La unidad dos, cuyo nombre es "2. Las Ecuaciones Diferenciales de primer orden", permite la integración de procesos estructurados de solución de ecuaciones diferenciales, que se equiparan con los procesos de solución de problemas en el contexto laboral.

Para el caso de la unidad tres, que versa en torno a la temática "3. Aplicaciones de las Ecuaciones Diferenciales de primer orden", se da un referente práctico al estudiante, acerca de la puesta en funcionamiento, de las ecuaciones diferenciales, es decir su aplicación y uso para la toma de decisiones en diversos campos del conocimiento.

\subsection{Análisis de la secuencia y desagregación de contenidos de acuerdo a la disciplina}

A continuación, se muestra la secuencia temática de la materia de ecuaciones diferenciales, sus diferentes subtemas y la duración (en horas), sugerida para la impartición de cada temática.

1. Las Ecuaciones Diferenciales

1.1. Principios de Modelado (3hrs 0min)

\subsubsection{Definición}

1.1.2. Clasificación

1.1.3. Soluciones (General y Particular)

1.1.4. Tipos de condiciones

1.2. Referente Gráfico (2hrs 0min)

1.2.1. Campos Direccionales

1.3. Teorema de Existencia y Unicidad (2hrs 0min)

2. Las Ecuaciones Diferenciales de primer orden

2.1. Diferentes formas de una ecuación diferencial de primer orden. (3hrs 0min)
2.2. Ecuaciones diferenciales de variables separables. (8hrs 0min)

2.2.1. Ecuaciones diferenciales con funciones homogéneas.

2.2.2. Otros tipos de ecuaciones reducibles a variables separables.

2.3. Ecuaciones Diferenciales Exactas y reducibles a exactas. (11hrs 0min)

2.4. Ecuaciones Diferenciales Lineales y reducibles a lineales (Bernoulli, Riccati y Clairaut). (9hrs 0min)

2.5. Solución numérica. (11 hrs 0min)

2.5.1. Método de Euler.

2.5.2. Euler mejorado

2.5.3. Runge Kutta.

3. Aplicaciones de las Ecuaciones Diferenciales de primer orden.

3.1. Modelación y Análisis de procesos de crecimiento y decrecimiento (variación). (7hrs 0min)

3.2. Otras aplicaciones de acuerdo a la Licenciatura. (14hrs 0min)

De acuerdo con la reflexión con respeto del contenido temático y la desagregación de los subtemas, se puede vislumbrar una posible área de mejora, como complemento a la formación del ingeniero industrial, en la formación de sus procesos cognitivos para la toma de decisiones. En este contexto, es prudente, la inclusión de la temática de "Transformada de Laplace", debido a que permite al estudiante, iniciar con la comprensión del abordaje de problemas desde un enfoque diferente (diferente espacio de soluciones), lo cual coadyuva en su proceso de razonamiento, ya que, al estar inmerso en el ambiente laboral, se contará con un referente que, cognitivamente, le permite abordar problemas desde diferentes contestos, enfoques o puntos de vista.

2.3 Relación entre las competencias genéricas y disciplinares con el objetivo general de la asignatura

El objetivo general de la asignatura consiste en: Crear y resolver modelos matemáticos, que involucren la variación en los sistemas y que ayuden a analizarlos para la y toma de decisiones.

\subsubsection{Competencias genéricas:}

\subsubsection{Competencia de Ciudadanía}

Permite la resolución de problemas en un contexto industrial con una base matemática, fomentando con ello el desarrollo del estudiante, coadyuvando en su formación profesional, con un impacto directo en la sociedad.

Competencia de Comunicación

Posibilita el desarrollar en los estudiantes la capacidad de la comunicación, para la comunicación de posibles soluciones a problemas con lo que se encuentren en un futuro contexto industrial o social, ayudando también, en la comunicación de las acciones producto de la toma de decisiones.

2.3.1.2 Competencia de Creatividad 
Aplicar la creatividad para detectar, formular y solucionar problemas de forma original e innovadora, a través de la integración de contenidos y mediante el uso de estrategias didácticas que generen el pensamiento divergente, problémico, investigativo, cooperativo, innovador, entre otras.

\subsubsection{Competencia de Formación}

La materia permite integrar los contenidos en diversas situaciones académicas, profesionales, sociales, productivas, laborales e incluso investigativas, creándose la posibilidad de aportar soluciones a problemas a través del empleo de métodos y procedimientos, con impacto en las organizaciones y en la sociedad.

2.3.1.4 Competencia de Liderazgo Colaborativo

El liderazgo colaborativo se ve reflejado en la identificación y desarrollo de ideas, proyectos de mejora, planes de acción, etcétera, con lo cual se, soporta la toma de decisiones en la organización a través de la formación de equipos de trabajos, liderados, en principio, por el ingeniero industrial.

\subsubsection{Competencia de Pensamiento Crítico}

La aplicación del pensamiento crítico permite llevar a cabo procesos de planeación, con la finalidad de resolver problemática al interior de las organizaciones y en la misma sociedad.

\subsubsection{Competencia de Uso de la Tecnología}

Las tecnologías de la información y la comunicación, se vislumbran como una herramienta para la toma de decisiones, a través de la concesión y expresión de los saberes adquiridos en el aula y puestos en práctica en los diferentes contextos de la vida cotidiana.

\subsubsection{Competencia Específica:}

2.3.2.1 Diseño, Implementación y Control de Condiciones de Trabajo Óptimas

A través de la materia, el estudiante adquiere las bases para diseñar las condiciones óptimas en el campo laboral y en para su rol en la sociedad, dada la estructuración metódica de procedimientos para la solución de problemas matemáticos (ecuaciones diferenciales lineales), que se equiparan al proceso de solución y toma de decisiones en las empresas y organizaciones.

\subsection{Evaluación de los saberes de competencias con relación a los temas, estrategias de enseñanza y aprendizaje.}

El desarrollo de competencias en el estudiante implica la cobertura, aprendizaje y evaluación desde diferentes ámbitos (Molina-Ruiz \& Rojano-Chávez, 2015). Como se menciona en Molina-Ruiz \& Rojano Chávez (2019), se pueden reconocer diferentes momentos de la evaluación y los diferentes enfoques de esta, por lo cual se debe resaltar la importancia de la evaluación en el ambiente de educación por competencias.

La evaluación es el proceso que tiene por objeto determinar en qué medida se han logrado unos objetivos previamente establecidos, lo cual supone un juicio de valor sobre la planeación establecida (UAEH, s.f.). Cabe destacar que la evaluación del aprendizaje, es uno de los aspectos importantes cuando se habla de los procesos de significación en el nuevo contexto de educación por competencias (Molina-Ruiz \& Rojano Chávez, 2019). Evaluar hace referencia a cualquier proceso por medio del que alguna o varias características de un estudiante, grupo de estudiantes, ambiente educativo, objetivos educativos, materiales, profesores, programas, etc., reciben la atención del que evalúa, se analizan y se valoran sus características y condiciones en función de unos criterios o puntos de referencia para emitir un juicio que sea relevante para la educación (Gimeno-Sacristán y Pérez-Gómez, 1996).

Por un lado, el propósito de la evaluación, es calificar el estudio y valorar el aprendizaje, además de contrastar resultados de los estudiantes, por otro, sirve para corregir, regular y mejorar el aprendizaje (Molina-Ruiz \& Rojano Chávez, 2019). Se pueden distinguir tres tipos de evaluación, la evaluación diagnóstica, formativa y sumativa.

La evaluación de los aprendizajes se encuentra estructurada en tres momentos:

1. Evaluación diagnóstica: La cual se lleva a cabo a través de estrategias informales, como lo es la observación y la exploración a través de preguntas a cerda de conceptos relacionado con el contexto de las ecuaciones diferenciales; estrategias formales, como lo es la prueba escrita con preguntas abiertas para la conceptualización de la materia y ejercicios matemáticos para la solución de ecuaciones diferenciales lineales.

2. Evaluación formativa: que se desarrolla a través de herramientas semiformales, como un problemario de ejercicios resueltos, y la integración de un portafolio de evidencias; herramientas formales, como lo es el examen escrito con ejercicios a resolver y el desarrollo de mapas conceptuales para generar la base teórica sobre las temáticas abordadas.

3. Evaluación sumativa: la cual se basa en estrategias semiformales, como el seguimiento y entrega de un portafolio de evidencias; además de contar con estrategias formales, es decir, una prueba global con ejercicios matemáticos.

\subsection{Crítica constructiva de la asignatura}

$\mathrm{Si}$ bien es cierto que el conocimiento matemático es relativamente estable a lo largo del tiempo también es cierto que el docente necesita mantenerse actualizado. De acuerdo con la visión de mediano y largo plazo de la universidad objeto de estudio, se pretende ser reconocida como una de las mejores universidades a nivel global, y posicionarse al nivel universidades como Harvard, MIT, Princeton o Cambridge, por lo cual es prudente que el docente inicie el uso de bibliografía en un segundo idioma, 
lo cual, en el corto plazo, le permite contribuir a la visión de mediano y largo plazo que posee la universidad objeto de estudio.

\subsection{Discusión teórica de la bibliografía con la postura personal sobre la asignatura}

Es importante mantener actualizada la bibliografía sugerida para la impartición de la asignatura, en la versión actual, se encuentra sugerido el uso de referencias tales como:

Zill, Dennis G. Ecuaciones Diferenciales con aplicaciones modelado (1a. Edición en español). México, Ed. Grupo Editorial Iberoamericano. 1997.

Marcus, Daniel A. Ecuaciones Diferenciales. México, Ed. CECSA. 2009.

Bronson, Richard. Y Costa G. Ecuaciones Diferenciales (3a. Edición). México, Ed. Mc Graw Hill, Serie Schaum. 2008.

Ayres, Frank Jr. Ecuaciones Diferenciales. México, Ed. Mc Graw Hill, Serie Schaum. 2009.

Lomen D. y Lovelock D. Ecuaciones Diferenciales a través de gráficas, modelos y datos (1a. Edición). México, Compañía Editorial Continental. 2000.

Golubitsky, M. Álgebra lineal y ecuaciones diferenciales, con uso de matlab. México:Thomson. 2011.

Las cuales son versiones publicadas en los años 1997, 2000, 2008, 2009 y 2011, con lo que se observa que la bibliografía tiene más de 8 años de publicada, con una sugerencia bibliográfica que supera los veinte años de publicación. Dado lo anterior se sugiere, una actualización bibliográfica, que incluya los siguientes títulos:

Agarwal, R. P., Hodis, S. \& O'Regan, D. (2019). 500 Examples and Problems of Applied Differential Equations. Problem Books in Mathematics, DOI: [10.1007/978-3-03026384-3].

García Guirao, J. L., Murillo Hernández, J. A. \& Periago Esparza, F. (Eds.). (2019). Recent Advances in Differential Equations and Applications. SEMA SIMAI Springer Series, DOI: [10.1007/978-3-030-00341-8].

Keskin, A. Ü. (2019). Ordinary Differential Equations for Engineers, DIO: [10.1007/978-3-319-95243-7].

Kolokoltsov, V. (2019). Differential Equations on Measures and Functional Spaces. Birkhäuser Advanced Texts Basler Lehrbücher, DOI: [10.1007/978-3-030-03377-4].

Quarteroni, A. \& Saleri, F (2006). Capítulo 7: Ecuaciones diferenciales ordinarias, Cálculo Científico con MATLAB y Octave, DOI: [10.1007/978-88-470-0504-4].

Struthers, A. \& Potter, M. (2019). Differential Equations, DOI: [10.1007/978-3-030-20506-5].

Zill, Dennis G. (2017) Ecuaciones Diferenciales con aplicaciones modelado, $10^{\text {a }}$ edición, ISBN 10: 6075194460, ISBN 13: 9786075194462, Editorial Cengage learning.

En este caso, el 71 \%de la bibliografía, contaría con una antigüedad de publicación de 1 año, mientras que se contaría con una referencia del año 2017 y otra, la más antigua, del año 2006.

\subsection{Propuestas de actualización}

A continuación, se presenta una propuesta de actualización para la materia objeto de estudio, que aborda dos características principales: la actualización o adecuación de contenidos y la actualización de la bibliografía sugerida para impartición de clase.

\subsubsection{Contenidos:}

2.7.1.1 Propuesta de ajuste del contenido temático Se propone agregar una cuarta unidad que aborde la temática de Transformada de Laplace, de la siguiente manera:

4.1 Introducción a la Transformada de Laplace

4.1.1 Transformada de Laplace

4.1.2 Propiedades de la Transformada de Laplace

4.1.3 Circunvolución y Solución de Ecuaciones Diferenciales Ordinarias

4.2 Ecuación de Laplace

4.2.1 Funciones armónicas

4.2.2 Soluciones de la Ecuación de Poisson

4.2.3 Soluciones Clásicas para Problemas de Valor de Frontera

4.2.4Solución en los Espacios de Sobolev y Besov

4.3 Aplicaciones para la Transformada de Laplace

4.3.1 Aplicaciones en Control Automático

4.3.2 Aplicaciones en Electricidad

4.3.2 Aplicaciones en Ingeniería de Control

4.3.3 Aplicaciones en Conducción de Calor y Problemas de Vibraciones

4.4 Tablas para la transformada de Laplace

4.4.1 Transformada de Laplace

4.4.2 Transformada Inversa de Laplace

2.7.1.2 Justificación

Dentro del campo laboral del ingeniero industrial, la solución de problemas en el contexto organizacional, requiere el abordaje de los problemas, desde perspectivas diferentes

2.7.1.3 Bibliografía específica sugerida para la propuesta de ajuste del contenido temático

4.1 Introducción a la Transformada de Laplace

Dyke, P. (2014). An Introduction to Laplace Transforms and Fourier Series. Springer Undergraduate Mathematics Series, DOI: [10.1007/978-1-4471-6395-4].

4.2 Ecuación de Laplace

Medková, D. (2018). The Laplace Equation, DOI: [10.1007/978-3-319-74307-3].

4.3 Aplicaciones para la Transformada de Laplace

Graf, U. (2004). Applied Laplace Transforms and zTransforms for Scientists and Engineers, DOI: [10.1007/978-3-0348-7846-3].

4.4 Tablas para la transformada de Laplace

Oberhettinger, F. \& Badii, L. (1973). Tables of Laplace Transforms, DOI: [10.1007/978-3-642-65645-3]. 


\subsubsection{Bibliografía}

La bibliografía sugerida, con el objetivo de actualizar la impartición de la asignatura objeto de estudio, es:

Agarwal, R. P., Hodis, S. \& O'Regan, D. (2019). 500 Examples and Problems of Applied Differential Equations. Problem Books in Mathematics, DOI: [10.1007/978-3-03026384-3].

Dyke, P. (2014). An Introduction to Laplace Transforms and Fourier Series. Springer Undergraduate Mathematics Series, DOI: [10.1007/978-1-4471-6395-4].

García Guirao, J. L., Murillo Hernández, J. A. \& Periago Esparza, F. (Eds.). (2019). Recent Advances in Differential Equations and Applications. SEMA SIMAI Springer Series, DOI: [10.1007/978-3-030-00341-8].

Graf, U. (2004). Applied Laplace Transforms and zTransforms for Scientists and Engineers, DOI: [10.1007/978-3-0348-7846-3].

Keskin, A. Ü. (2019). Ordinary Differential Equations for Engineers, DIO: [10.1007/978-3-319-95243-7].

Kolokoltsov, V. (2019). Differential Equations on Measures and Functional Spaces. Birkhäuser Advanced Texts Basler Lehrbücher, DOI: [10.1007/978-3-030-03377-4].

Medková, D. (2018). The Laplace Equation, DOI: [10.1007/978-3-319-74307-3].

Oberhettinger, F. \& Badii, L. (1973). Tables of Laplace Transforms, DOI: [10.1007/978-3-642-65645-3].

Quarteroni, A. \& Saleri, F (2006). Capítulo 7: Ecuaciones diferenciales ordinarias, Cálculo Científico con MATLAB y Octave, DOI: [10.1007/978-88-470-0504-4].

Struthers, A. \& Potter, M. (2019). Differential Equations, DOI: [10.1007/978-3-030-20506-5].

Zill, Dennis G. (2017) Ecuaciones Diferenciales con aplicaciones modelado, $10^{\mathrm{a}}$ edición, ISBN 10: 6075194460, ISBN 13: 9786075194462 , Editorial Cengage learning.

\section{Conclusión}

La importancia de la educación superior para el desarrollo de los pueblos, exige al catedrático, adentrarse en proceso de reflexión profunda, a cerca de su quehacer docente. En específico, es de vital importancia, reflexionar acerca de las asignaturas impartidas, con el propósito de incidir positivamente en la mejora de los contenido o estructura de las materias impartida, propiciando un ambiente de mayor competencia para los estudiantes de los cuales es guía.

En el caso particular de la asignatura de ecuaciones diferenciales, se encuentran dos posibles áreas de mejora, la inclusión de la temática de transformad de Laplace, que considera la transformación del espacio de soluciones $(\mathrm{x}, \mathrm{y})$ al espacio de soluciones $(\mathrm{s}, \mathrm{t})$. Además de la actualización de la bibliografía sugerida parala impartición de clase, la cual se observan versiones publicadas en los años 1997, 2000, 2008, 2009 y 2011, incluyéndose una sugerencia bibliográfica que supera los veinte años de publicación.

\section{Referencias}

[1] Adhikari, S. (2019). The 10 oldest ancient civilizations that have ever existed, Ancient History Lists, URL: [https://www.ancienthistorylists.com/ancient-civilizations/10-oldestancient-civilizations-ever-existed/].

[2] Neugebauer, O., (1969). Exact Sciences in Antiquity, Dover Publications, Inc., Segunda Edición.

[3] Seidenberg, A. (1978). The origin of mathematics, Archive for history of exact sciences, 18(4), 301-342, DOI: [https://doi.org/10.1007/BF00348435], URL: [https://link.springer.com/article/10.1007\%2FBF00348435].

[4] Martzloff, J.C. (1997). A History of Chinese Mathematics, DOI: [10.1007/978-3-540-33783-6], [https://www.springer.com/gp/book/9783540337829].

[5] Boyer, C.B. \& Merzbach U.C. (2011). Capítulo 2: Ancient Egypt, En: A History of Mathematics, 3rd Edition, ISBN: 978-0-470-52548-7.

[6] Quarteroni, A. \& Saleri, F (2006). Capítulo 7: Ecuaciones diferenciales ordinarias, En: Cálculo Científico con MATLAB y Octave, DOI: [10.1007/978-88-470-0504-4].

[7] Molina-Ruiz, H.D. \& Rojano Chávez, S.M. (2015). Estrategia de desarrollo socio-emocional en alumnos de bachillerato, para fortalecer la formación del estudiante, en el nuevo paradigma de educación por competencias, REVISTA CoPEI, APRENDER A APRENDER INNOVANDO, 2(3), pp. 155-162, ISSN: 2395 - 8375, URL: [http://www.cipei.org/revista/2do-congreso/pdf/Revista-3.pdf].

[8] Molina-Ruiz, H.D. \& Rojano-Chávez, S.M. (2019). Cap. 11: Evaluación de los objetos de aprendizaje en la formación por competencias, En: Tecnologías aplicadas a la investigación, Editores: Tomás FontainesRuiz \& Jorge Maza-Cordova, ISBN: 978-9942-8772-0-8, Ecuador: Ediciones RISEI, URL: [tecnologiasparainvestigar.risei.org].

[9] UAEH (s.f.). Modelo educativo de la UAEH, Universidad Autónoma del Estado de Hidalgo, URL: [https://www.uaeh.edu.mx/docencia/docs/modelo_educativo_UAEH.p df].

[10] Gimeno-Sacristán, J. \& Pérez-Gómez, A. I. (1996). La evaluación en la enseñanza, Comprender y transformar la enseñanza, Madrid, pp. 334352. 